\section{Comparison of Clinically Significant Infection Rates Among Prehospital Versus In-hospital Initiated Intravenous Lines}

\author{
*Robert Levine, MD, Daniel W. Spaite, MD, \\ FACEP, Elizabeth A. Criss, RN, Terence D. \\ Valenzuela, MD, FACEP, A. Lawrence Wright, \\ PhD, Harvey W. Meislin, MD, FACEP
}

Arizona Emergency Medicine Research Center, Health Services Research Program, University of Arizona, Tucson, Arizona

Purpose: To compare the risk of infection for intravenous lines (IV) placed in the prehospital versus in-hospital setting in a mid-sized EMS system.

Methods/Design/Setting: A retrospective analysis of all IV-site infections among patients admitted to ward beds from a University Hospital Emergency Department (ED) in 1992. During the study, the hospital's infection control team conducted daily ward rounds and a surveillance of all wound and blood cultures. Those patients with signs and/or symptoms consistent with the [US]Centers for Disease Control and Prevention guidelines for skin and soft tissue infection were reported to the responsible medical team. Infections were documented based on consensus opinion between the infection control team and the physicians responsible for the care of the patient. Those IVs placed in the prehospital phase of care were identified by electronic retrieval from the prehospital database.

Results: A total of 3,185 patients who had a prehospital or in-hospital IV placed were admitted from the ED. Of these, 859 IVs were placed prehospital (27\%) and 2,326 were initiated in-hospital (73\%). There was one infection in the prehospital study cohort and four in the inhospital group. This yields an infection rate of 0.0012 among prehospital patients and 0.0017 among in-hospital patients ( $p=0.591$; Fisher's Exact Test).

Conclusions: This study yielded exceptionally low infection rates in both cohorts. No clinically or statistically significant increase in the risk of infection among prehospital or in-hospital initiated lVs was identified. These data do not confirm the findings of a previous study that noted an increased risk of infection in patients with prehospital-initiated IVs. The broad clinical criteria used in the prior report, differences in patient populations, or differences in prehospital or in-hospital care mayaccount for this.

\section{Quality Indicators for Emergency Medical Services: The Paramedics' Perspective}

\author{
*Miles D. Greenberg, MD, Vincent K. Mosesso, \\ $M D$, Theodore R. Delbridge, MD, Ronald K. Roth, \\ $M D$, Herbert G. Garrison, MD, MPH, Paul M. \\ Paris, MD, William R. Miller, EMT-P \\ Division of Emergency Medicine, University of Pittsburgh, \\ Pittsburgh, Pennsylvania
}

Objective: Out-of-hospital emergency medical services (EMS) need relevant and measurable indicators of quality. According to the principles of total quality management (TQM), front-line workers who provide service directly to the customer are integral to the process of defining quality. The objective of this investigation was to obtain from paramedics - those members of the EMS team closest to the patient-their views about what constitutes quality in EMS.

Methods: During regular education sessions, paramedics from a large municipal EMS system, were given a presentation on TQM. The paramedics then were assigned to focus groups of five to seven members, asked to identify five quality indicators, and decide how they should be measured.

Results: A total of 102 paramedics participated in the focus groups. Fifteen separate EMS quality indicators were identified: 1) patient satisfaction; 2) patient outcome; 3) EMS crew satisfaction; 4) partner performance; 5) paramedic wellness/occupational injuries; 6) EMS cost-effectiveness; 7) equipment practicality; 8) managerial satisfaction; 9) dispatch accuracy; 10) call quality; 11) response times; 12) complaints; 13) crew and equipment appearance; 14) public confidence; and 15) innovations/research. Ways proposed to measure the quality indicators included: 1) surveys of patients and families; 2) surveys of paramedics; 3) tracking unsolicited comments; 4) tracking compensation awards; 5) tracking media coverage; 6) tracking paramedic attrition rates and absenteeism; 7) tracking grievances; 8) determining cost-benefit ratios; and 9) outcome studies.

Conclusions: From the perspective of the paramedics studied, indicators of EMS quality differ from traditional EMS quality assurance measures, e.g., success rates for endotracheal intubation and intravenous cannulation. TQM theory predicts that the paramedic-generated indicators are more likely to reflect "true" system quality. Future studies should investigate the applicability of these indicators to EMS quality management. 